Revisión/Review

\section{MODELOS EXPLICATIVOS DE CARIES DE LA INFANCIA TEMPRANA}

\section{EXPLANATORY MODELS OF CARIES OF EARLY CHILDHOOD}

\author{
Fierro Monti $\mathrm{C}^{1}$, Pérez Flores A. \\ ${ }^{I}$ Departamento de Pediatría Bucal, Universidad de \\ Concepción, Chile
}

\section{Resumen}

La caries de la infancia temprana (CIT) es una enfermedad crónica altamente prevalente en niños, siendo un problema de salud pública, porque afecta una población considerada vulnerable. La construcción de modelos, en la planificación y evaluación de intervenciones en salud, resulta una herramienta valiosa, siendo frecuentemente utilizados para descubrir su etiología así como predecir el curso de la enfermedad. Se han presentado 11 modelos explicativos de la caries de la infancia temprana construidos en diversas poblaciones mundiales, que presentan similitudes y diferencias propias de los estudios y sus poblaciones infantiles.

Objetivo: Identificar a través del tiempo, los diferentes tipos de modelos explicativos gráficos de la CIT

Método: La búsqueda bibliográfica se realizó en MEDLINE, EMBASE, LILACS, SciELO, Science Direct, IBECS, Cochrane y CENTRAL conjugando los términos "early childhood caries", "explanatory models", "risk models", " graphic models", "conceptual models" con límites de idioma inglés y español, publicados entre 1995 у 2017.

Resultados: Se presentan 11 modelos gráficos explicativos de CIT realizados en EUA, Reino Unido, Suecia, China, Escocia, Chile, y Brasil.

Conclusión: Los modelos teóricos son construidos a fin de explicar la causalidad de los procesos biológicos, psicológicos y sociales que intervienen en el desarrollo de la enfermedad. Los modelos exlicativos presentados dan cuenta de las similitudes y diferencias presentes a edades tempranas en la población estudiada para dar causalidad y predicción a la caries.

PALABRAS CLAVE: modelos explicativos, modelos de riesgo de caries de la infancia temprana.

\begin{abstract}
Early childhood caries (CIT) is a highly prevalent chronic disease in children, being a public health problem because it affects a population considered vulnerable. The construction of models, in the planning and evaluation of health interventions, is a valuable tool, being frequently used to discover its etiology as well as to predict the course of the disease. We have presented 11 explanatory models of early childhood caries constructed in diverse world populations, which present similarities and differences characteristic of studies and their children populations.

Objective: To identify over time, the different types of graphic explanatory models of the CIT.

METHODS: The literature search was carried out in MEDLINE, EMBASE, LILACS, SciELO, Science Direct, IBECS, Cochrane and CENTRAL, combining the terms "early childhood caries", "explanatory models", "risk models", "graphic models", "conceptual models "with limits of English and Spanish language, published between 1995 and 2017.

Results: 11 CIT explanatory graphic models are presented in USA, UK, Sweden, China, Scotland, Chile, and Brazil.

Conclusion: The theoretical models are constructed in order to explain the causality of the biological, psychological and social processes involved in the development of the disease. The explicative models presented account for the similarities and differences present at early ages in the populations studied to give causality and prediction to caries.
\end{abstract}

KEY WORDS: explanatory models, risk models for early childhood caries.

\section{Introducción}

La Caries de la Infancia Temprana (CIT) es una enfermedad crónica altamente prevalente en niños. Esta enfermedad continúa siendo un problema de salud pública, ya que afecta a una población vulnerable ${ }^{1}$. La CIT es una enfermedad mulifactorial que junto a otras como cáncer, enfermedades-cardiovasculares, siquiátricas y diabetes; dónde es difícil elegir las formas de prevención, promoción o atención en salud ${ }^{1}$.

La construcción de modelos en la planificación y evaluación de intervenciones en salud, resulta una herramienta valiosa, siendo frecuentemente utilizados para predecir su evolución. En el contexto de la salud pública, son construidos a 
fin de explicar la causalidad de los procesos biológicos, psicológicos y sociales que intervienen en el desarrollo de la enfermedad ${ }^{2}$. Los primeros modelos de CIT se basaron principalmente en las teorías psicológicas, considerando el comportamiento de las personas en relación a la salud ${ }^{3}$. Los factores sociales, conductuales y psicológicos, en conjunto con los factores biológicos permitieron en estos primeros modelos, aumentar la predicción de la CIT ${ }^{4,5}$. Sin embargo, estos fueron criticados por su falta de consideración del medio ambiente como otro factor de riesgo. En modelos más recientes se incluyen los determinantes socioeconómicos y ambientales de la salud, permitiendo su transferencia en la práctica clínica, porque contemplan las influencias sociales, políticas y ambientales ${ }^{6,7}$.

El objetivo de este estudio fue una revisión de la literatura científica y la identificación a través del tiempo de los diferentes tipos de modelos explicativos de CIT en diferentes contextos geográficos del mundo.

\section{Métodos}

\section{Búsqueda bibliográfica}

Se realizó en MEDLINE, EMBASE, LILACS, SciELO, Science Direct, IBECS, Cochrane y CENTRAL conjugando los términos "early childhood caries", "explanatory models", "risk models", "graphic models", "conceptual models" con límites de idioma inglés y español, publicados entre 1995 y 2017. Además, se amplió la búsqueda a las referencias de los trabajos seleccionados y se complementó con otras fuentes de información consideradas relevantes.

\section{Resultados}

De la revisión se identificaron los modelos explicativos de CIT que se muestran en la Tabla 1.
Tabla 1. Resumen de Modelos para caries de la infancia Temprana con orden cronológico

\begin{tabular}{|c|c|c|c|}
\hline $\begin{array}{ll}\text { Tipo de } \\
\text { modelo }\end{array}$ & Descripción general & Autor & Año, Origen \\
\hline \multirow[t]{2}{*}{$\begin{array}{l}\text { Causal } \\
\text { /explicativo }\end{array}$} & $\begin{array}{l}\text { Modelo causal } \\
\text { multidimensional de } \\
\text { desarrollo de caries } \\
\text { en niños } \\
\text { preescolares de } \\
\text { estrato económico } \\
\text { bajo }\end{array}$ & Litt et al & 1995, EUA \\
\hline & $\begin{array}{l}\text { Modelo explicativo } \\
\text { de inequidades en } \\
\text { salud de la caries } \\
\text { dental en la infancia }\end{array}$ & Pine et al & $\begin{array}{l}\text { 2004, } \\
\text { Reino Unido }\end{array}$ \\
\hline \multirow[t]{4}{*}{ Multinivel } & $\begin{array}{l}\text { Modelo conceptual } \\
\text { de los determinantes } \\
\text { de la salud bucal de } \\
\text { los niños }\end{array}$ & $\begin{array}{c}\text { Fisher-Owens } \\
\text { et al }\end{array}$ & 2007, EUA \\
\hline & Cariograma & $\begin{array}{c}\text { Holgerson et } \\
\text { al }\end{array}$ & 2009, Suecia \\
\hline & $\begin{array}{l}\text { Modelo } \\
\text { conceptual multi } \\
\text { nivel de salud bucal } \\
\text { en niños pequeños }\end{array}$ & Bramlett et al & 2010, EUA \\
\hline & $\begin{array}{l}\text { Modelo de evalución } \\
\text { de riesgo }\end{array}$ & Gao et al & 2010, China \\
\hline \multirow{5}{*}{$\begin{array}{l}\text { De } \\
\text { evaluación } \\
\text { de riesgo }\end{array}$} & Cambra & $\begin{array}{c}\text { Domejean et } \\
\text { al }\end{array}$ & 2011, EUA \\
\hline & $\begin{array}{l}\text { Modelo de } \\
\text { evaluación de riesgo } \\
\text { de caries }\end{array}$ & $\begin{array}{c}\text { Macritchie et } \\
\text { al }\end{array}$ & $\begin{array}{l}2012, \\
\text { Escocia }\end{array}$ \\
\hline & $\begin{array}{l}\text { Modelo conceptual } \\
\text { unificador }\end{array}$ & Seow et al & 2012; EUA \\
\hline & $\begin{array}{l}\text { Modelo simple } \\
\text { predictivo de caries } \\
\text { de la infancia } \\
\text { temprana. }\end{array}$ & Fierro y col & 2014; Chile \\
\hline & $\begin{array}{l}\text { Modelo Teórico } \\
\text { Jerárquico de } \\
\text { factores asociados a } \\
\text { la polarización de } \\
\text { CIT }\end{array}$ & Nunes et al & 2014, Brasil \\
\hline
\end{tabular}




\section{Modelo de Litt y otros}

En el año 1995, Litt y sus colaboradores ${ }^{8}$ formula un modelo para caries basado en los factores que impactaban en la salud oral, como biológicos, cognitivos, conductuales y demográficos. En los factores biológicos consideró que el nivel de $S$. mutans era un fuerte contribuyente en el desarrollo de la caries, en conjunto con Lactobacillus sp. y el fluoruro del medio bucal. En este primer modelo se encontró que el estrés (entendido como tensión emocional) de vida estaba relacionado con la presencia de CIT; y se estableció que los padres que lograban estar sometidos a situaciones de vida con mayor estrés tenían niños con menor presencia de caries. Una situación, en que estas personas son "hipervigilantes" y se comportan de una manera más protectora hacia sus hijos. En este modelo los hábitos, la alimentación e higiene, el consumo de azúcares, el cepillado dental y, el uso del biberón se reconocieron también como factores importantes en el desarrollo de caries. En la aplicación del modelo a distintas realidades se observó que la inclusión de la variable étnica fue motivo de discenso en los resultados. La distinción más importante se presentó entre los hispanos y otros grupos, con mayor diferencia entre blancos y negros, sin embargo no se determinó fehacientemente por qué los hispanos presentan menores niveles bacterianos. Utilizando el modelado causal se investigaron predictores directos e indirectos, tasas de caries y de $S$. Mutans en niños de bajos ingresos. Este modelo indica que el desarrollo de caries es más complejo de lo que generalmente se conceptualiza en la literatura hasta esa época; sin embargo actualmente se conoce que ninguna de las variables iniciales mencionadas, incluido el conocimiento sobre salud bucal, tiene una asociación directa con el ceo en las primeras etapas del desarrollo de caries $^{8}$.

\section{Modelo de Pine y otros}

En el avance de los modelos explicativos, Pine et al, $2004{ }^{9}$ describen que las áreas críticas en el progreso de la caries son los aspectos sociales y culturales generados durante el desarrollo del niño. Donde se mencionan el estrés familiar, el uso de los servicios de salud, la nutrición, el acceso a fluoruro del agua (bebida), el hábito de consumo de azúcares, la composición y actividad de la microflora oral, el reconocimiento de los efectos conductuales y biológicos en la salud. Hasta este estudio las investigaciones publicadas habían examinado las asociaciones entre los factores de riesgo y el desarrollo de la caries en forma transversal y longitudinal ${ }^{10,11}$. Sin embargo, poco se conocía de las interacciones entre los impactos moleculares y psico-sociales particularmente, dentro y entre los niños con diversidad étnica o con deficiencias de salud bucal que devienen de las diferencias de culturas, propias de cada nación en el contexto mundial. Estos autores establecieron la relación entre los factores de riesgo y el desarrollo de la caries de la primera infancia en un modelo explicativo inicial. Los autores destacaron las principales influencias en el desarrollo de riesgo de caries. Establecieron que la calidad del mineral observado en dientes deciduos depende de un desarrollo adecuado durante el período intrauterino; y que la nutrición materna es un factor que impacta en la nutrición general y el desarrollo del niño. Por otra parte, los autores consideraron que la hipoplasia, de los dientes deciduos, es un biomarcador de una serie de perturbaciones sistémicas, incluyendo la desnutrición intrauterina y las enfermedades infecciosas en el primer año de vida. En este modelo, los impactos ecológicos incluyeron los impactos ambientales inmediatos que dan lugar al estrés materno.

Además, evaluaron a cada niño en un grupo de alto riesgo de caries dentro de una población, indicando características principales como la pobreza y la diversidad étnica. Estas particularidades de los niños fueron asociadas con el desarrollo de caries tanto dentro, como entre los diversos grupos de la población, permitiendo, a los autores, establecer que la condición de la pobreza podría actuar como un componente moderador independiente del riesgo. En esta etapa del conocimiento sobre la caries, hubo mayores similitudes que diferencias entre los grupos étnicos ${ }^{12}$ y la mayor diferencia estuvo dada por la condición de pobreza. Los estudios consideraron que era importante tener en cuenta las personas en su propio entorno, la comprensión de sus 
creencias, actitudes, dieta, los comportamientos en salud, las estructuras sociales y ambientales que son en definitiva los factores que mayormente promueven la desigualdad frente a la condición de salud ${ }^{13,14}$.

\section{De Fisher-Owens}

El modelo Fisher-Owens et al, $2007^{15}$ incorpora influencias de 5 dominios principales: los factores genéticos-biológicos, el medio ambiente social, y el físico, los hábitos en salud $\mathrm{y}$ los cuidados médicos $\mathrm{y}$ odontológicos. El modelo reconoce la presencia de un interjuego complejo de los factores causales e incorpora el tiempo de evolución de las enfermedades bucales, como la caries. Aunque algunas de las relaciones descritas involucran los factores conocidos que afectan la salud física en general, aún no se han estudiado por separado los que tienen un impacto sobre la salud bucal. Existen coincidencias suficientes entre los factores que afectan a la salud general y la salud bucal, en particular, por lo que su inclusión en un modelo conceptual es razonable, incluso si aún no se han identificado efectos específicos sobre la salud bucal. Se incluye un elemento de tiempo, reconociendo que la salud bucal de los niños es dinámica; cada niño tiene una trayectoria de desarrollo único. Por último, se encuentra implícito en el modelo el concepto de la vulnerabilidad y la capacidad de recuperación ${ }^{15}$.

\section{Cariogram}

Holgerson et al., 2009 ${ }^{16}$ diseñaron un estudio para validar los perfiles de riesgo de caries evaluados con el modelo Cariogram en comparación con el desarrollo real de caries en los niños en edad preescolar. En el Cariogram original, se califican nueve parámetros diferentes que se introducen en el programa computacional. Para el Cariograma modificado, se utilizaron sólo siete parámetros: 1) la experiencia de caries 2) el recuento de Streptoccocus mutans, 3) las enfermedades o medicamentos pertinentes, 4) la frecuencia de consumo de azúcar, 5) la higiene bucal, 6) el uso de fluoruros, y 7) el criterio clínico.

En relación a la información sobre saliva, en el estudio desarrollado por los autores, no se incorporó el dato dela velocidad de flujo por minuto de ésta. En la investigación se observó en el grupo con alto riesgo de desarrollo de caries, que experimentaron la aparición de la mayoría de las lesiones nuevas. Con este modelo la sensibilidad y los valores predictivos negativos fueron del 90\%, mientras que la especificidad y los valores predictivos positivos fueron de alrededor del 50\%. El porcentaje de niños clasificados correctamente como verdaderos positivos y verdaderos negativos fue $63 \%$. La calidad de este estudio fue calificada como regular ${ }^{17}$.

\section{De Bramlett y col.-Conceptual}

Bramlett et al, 2010 ${ }^{18}$ prueban empíricamente un modelo conceptual de múltiples niveles de salud bucal en niños pequeños, con datos de la encuesta nacional realizada en EEUU (2003). El modelo incorpora 22 dominios de la salud bucal infantil en cuatro niveles: los niños, la familia, la comunidad y el estado; donde los factores incluidos en los cuatro niveles se asociaron con la probabilidad de que los padres calificaran la salud bucal de sus hijos, como regular o mala. De los 22 dominios, 15 contenían factores asociados significativamente con la salud bucal de los niños. A nivel estatal, el acceso al agua fluorurada se asoció significativamente con la salud bucal favorable para los niños, al igual que la edad y el origen étnico. Sin embargo, se observaron una serie de correlaciones significativas que son susceptibles de intervenciones a nivel de la Salud Pública, como es el acceso a agua fluorada, la familia la cohesión del barrio, y los comportamientos positivos para la salud paterna.

Las limitaciones del modelo incluyen el uso de datos longitudinales y la recolección de datos sobre los indicadores más próximos para algunos dominios, como la dotación genética. La variable dependiente resultó una medida subjetiva del informe paterno de la condición dentaria del niño; la cual no fue validada utilizando exámenes clínicos dentales. Por último, aunque los autores fueron capaces de examinar un gran número de factores explicativos, hubo variables que fueron identificadas en el modelo conceptual, pero cuyos datos no estuvieron disponibles. Algunos 
dominios sólo fueron medidos por proximidad como el apoyo social, a nivel familiar y a nivel del niño: el comportamiento y las prácticas de salud. ${ }^{18}$

\section{Gao y colaboradores}

Gao et al., 2010 ${ }^{19}$ construyeron varios modelos de evaluación de riesgos, basados en componentes bio-psico-sociales en diversos entornos. El estudio lo realizaron aplicando un cuestionario, exámenes bucales y marcadores biológicos, como saliva, componentes microbiológicos y $\mathrm{pH}$. Para la predicción de un "incremento de caries en un año", los modelos de detección que no incorporaron las pruebas biológicas consiguieron una sensibilidad / especificidad del 82\% / 73\%; mientras que aquellos que incluyeron pruebas biológicas lograron una sensibilidad / especificidad del $90 \% / 90 \%$.

Para la identificación de niños con alta carga de caries (línea de base ceod> 2), el modelo comunitario de detección, que sólo requiere un cuestionario, alcanzó una sensibilidad/especificidad del 82\% / 81\%. Estos modelos evaluados por los investigadores resultaron herramientas prometedoras para el control de la caries en relación a costo, efectividad y planes de tratamiento ${ }^{19}$.

\section{Boyce y otros}

Boyce et al., $2010^{20}$ investigaron la relación entre CIT y las desigualdades sociales, a través de recuentos de UFC/ml, concentración de cortisol en saliva (marcador de estrés y ansiedad) y el índice de $\mathrm{CPO} / \mathrm{ceo}$. A partir de los resultados de su estudio, estos autores, extrajeron cinco conclusiones principales: a) aproximadamente un 50\% de los niños habían desarrollado caries dental con 5 años de edad; b) la familia sometida a estrés social produce una activación basal de la vía hipotalámicapituitaria-adrenal en el niño, relacionando este hecho con un mayor recuento de bacterias cariogénicas bucales; c) la presencia de bacterias cariogénicas y de cortisol en saliva se observaron asociados de modo independiente con el desarrollo de caries; d) el riesgo más alto de presencia de caries dental se observó en niños con alto niveles de cortisol en saliva y de bacterias cariogénicas en boca; y e) la presencia de cortisol se asoció con una superficie de esmalte más delgada, y por lo tanto más vulnerable a la caries dental.

Boyce et al., 2010 propusieron un modelo conceptual social y psicobiológico en relación a la incidencia de caries; con dos vías biológicamente posibles y distintas pero que interactúan. La hipótesis sobre la que basan este modelo es que los factores de estrés de la familia y los cambios relacionados que éste produce en la biología oral, podrían explicar, todo o en parte, las diferentes condiciones de salud bucal en niños con familias socioeconómicamente vulnerables. En el esquema se muestra la propuesta teórica que relaciona las condiciones socioeconómicas y biológicas desfavorables con la presencia de caries $^{20}$.

\section{Cambra}

Domejean et al, 2011 ${ }^{21}$ evaluaron retrospectivamente la validez de CAMBRA (el manejo de la caries por la filosofía de la evaluación de riesgo de caries, propugnada por la Asociación Dental de California) como modelo de evaluación de riesgo de caries, basado en la relación que existe entre la caries observada en el tiempo presente y que permite determinar su valor predictivo para la caries futura. El estudio llegó a la conclusión de que "la cavitación visible", "la penetración de caries en la dentina en forma radiográfica", y "las lesiones del esmalte interproximal 0 radiotransparencias" se relacionaron con el riesgo general de caries, al inicio del estudio. De los pacientes evaluados como extremos y de alto riesgo, $88 \%$ y $69,3 \%$ desarrollaron nuevas caries en el primer seguimiento. No se observó asociación entre los "manchas blancas" en el seguimiento y el riesgo basal de caries. No se proporcionaron los resultados relacionados con la sensibilidad y la especificidad de la predicción, y la falta de ajuste estadístico para los factores importantes de confusión que también podrían haber jugado un papel en el desarrollo de nuevas lesiones de caries es una de las principales limitaciones de este estudio. La calidad de este estudio fue calificado como pobre ${ }^{17}$. 


\section{De Dundee}

MacRitchie et al, $\mathbf{2 0 1 2}^{22}$ desarrollaron un modelo de evaluación de riesgo de caries en la ciudad de Dundee, Escocia. Estos autores realizaron el estudio utilizando un detector de interacción automática Chi Cuadrado que permite generar modelos para predecir el riesgo en niños de 4 años de edad. Mediante esta metodología los investigadores construyeron 4 modelos de riesgo (Fig. 5) a partir de datos de evaluación de riesgos recogidos al año de vida.

Dos de los modelos de riesgo incluyeron cualquier grado de riesgo de caries $(n=697$, COPD> 0) en tanto los otros dos contemplaron un alto riesgo de caries $(n=784$, COPD $>3)$ dependiendo del nivel de detección de caries: d1 (esmalte y dentina) o d3 (dentina solamente).

De los modelos obtenidos el que presentó mejor sensibilidad y especificidad fue el modelo de alto riesgo de caries (análisis CHAID) a nivel d3 de detección; el cual tuvo una sensibilidad del $65 \%$ y una especificidad del $69 \%$.

\section{Unificador de Riesgo de CIT}

A partir de la revisión de la literatura, Seow et al, $\mathbf{2 0 1 2}^{23}$ propone un modelo unificador que reúne factores maternos e infantiles que se asocian comúnmente con la CIT. Se propone como centro del modelo a la madre, que generalmente proviene de un entorno en desventaja social, nivel socioeconómico bajo y un bajo nivel de educación. Donde la madre no cree que los dientes de los niños sean importantes, que su hijo sea susceptible a presentar CIT; y que la CIT sea una enfermedad grave o que sea necesario o factible un tratamiento.

La falta de recursos de la comunidad y el hogar son comúnmente asociados con el estrés materno psicológico, la depresión y la ansiedad. En conformidad con el malestar psicológico, una madre puede recurrir a estrategias de crianza ineficaces y esto puede ser asociado con un sentido reducido de la eficacia en la crianza. También es probable que ella sea muy dura o muy permisiva e incoherente en la aplicación de reglas de convivencia. Las consecuencias de este tipo de crianza disfuncional son los niños que no cumplen con la conducta deseable en relación a la salud dental, tal como negarse a cooperar con el cepillado de dientes. Además, los niños con temperamento difícil pueden aumentar aún más el estrés psicológico en los padres y empeorar el comportamiento disfuncional de éstos. Una madre con un bajo nivel educativo y falta de conocimiento de salud dental muestra patrones de comportamiento que ponen su propia dentadura y la de su hijo en riesgo, tales como: la negligencia y la mala higiene bucal, la ingesta frecuente de bocadillos, la ingesta de alimentos con azúcar y la alimentación inapropiada con biberón azucarado. Estos comportamientos son propensos a resultar en altas cargas de bacterias cariogénicas que conducen a incrementar el riesgo del niño a desarrollar CIT. Se observa que, un niño en un ambiente con desigualdades sociales en el sistema de salud; es probable que esté en mayor riesgo de hipoplasia de esmalte, debido a una mayor predisposición a condiciones perinatales y neonatales, como la prematuridad al nacer. La hipoplasia del esmalte aumenta aún más el riesgo de los dientes a desarrollar CIT.

Se deduce que los vínculos entre los factores socioeconómicos, culturales y psicológicos con la presencia de CIT son complejos y en ocasiones se superponen. Se ejemplifica a una madre de bajo nivel socio económico con probables tensiones familiares, económicas y de otra índole, dónde es difícil determinar la contribución relativa de estas tensiones a la angustia psicológica derivada de la maternidad en general. Por otra parte, las vías causales complejas y los procesos que son propensos a estar involucrados no han sido completamente aclarados, y los autores informan que el actual modelo tendrá que ser aún más refinado con más evidencia clínica disponible. Es probable que una comprensión más profunda de estos enlaces tenga que venir de la investigación cualitativa, sobre todo en aspectos como las creencias y las actitudes maternas. Por lo tanto, aunque se necesitará evidencia de algunos de los enlaces internos que se establezcan, el actual modelo conceptual intenta proporcionar un marco útil que conecta los factores ambientales, sociales, psicológicos y conductuales que intervienen en la CIT. El modelo puede ser empleado para considerar las variables psicosocial materna, la crianza de los 
hijos, y el hijo como mediadoras en la investigación sobre las diversas vías etiológicas de la CIT $^{23}$.

\section{Fierro C y otros}

Se ha propuesto un modelo donde la higiene bucal, el consumo de azúcar, el sexo masculino, y el comportamiento difícil son los principales factores para predecir la presencia de $\mathrm{CIT}^{24}$. En este modelo, que es más sencillo que los elaborados por otros autores, tanto las situaciones de estrés que vive la madre y las que se generan en el hogar, influyen negativamente en el comportamiento del niño y en consecuencia permiten mayor probabilidad de desarrollar CIT. Estas variables comportamentales tuvieron una magnitud, medida en Odd Ratios, de igual peso que la higiene bucal y la ingesta de azúcar. Generalmente, los modelos propuestos de CIT en la literatura son muy complejos y no son de fácil aplicación a nivel de atención primaria de salud $^{9,22}$. El enfoque de los autores se basa en la identificación de las principales variables que se pueden supervisar fácilmente en la atención primaria de salud. Cuando los modelos de riesgo incluyen unas pocas variables, se puede activar la proyección a un gran número de niños. Petersen considera que ciertos patrones de comportamiento o estilos de vida influyen en los resultados a través de los procesos fisiológicos, y son solo algunos factores de riesgo sobre los que un individuo tiene, al menos, algo de control ${ }^{25}$. En esta investigación un porcentaje importante de las familias eran de clase media-baja, es decir era una población socioeconómicamente vulnerable. Además las características de comportamiento de los niños que fueron significativas, especialmente en los varones, eran la agresividad y el miedo entre otras. Todas relacionadas con el estrés en el hogar y de la madre, y asociadas significativamente a la presencia de caries $(\geq$ $4)^{24}$.

\section{Nunes y otros, 2014}

Emplearon ${ }^{26}$ un modelo teórico jerárquico con los 8 factores (bloques) siguientes: 1) edad de niño; 2) socioeconómicos/demográficos; 3) el pasado de caries de la madre (si la madre ha o no recibido tratamiento por caries); 4) el estado de salud y hábitos del niño; 5) alimenticios; 6) datos de higiene oral del niño; 7) Índice de placa bacteriana visible; y 8) nivel de contaminación de SM salival.

La edad se consideró como un factor potencialmente confundente, por tanto fue añadida como una variable continua. El segundo-bloque incluyó variables socioeconómicas y demográficas tales como la edad de la madre, años de educación materna, ingreso familiar, quien se quedó en casa con el niño cuando no estaba en el tratamiento ese día (madre/otros), y si la madre o cuidador recibieron información sobre la salud oral. En el quinto-bloque, las prácticas alimenticias se incluyeron, suponiendo que estas variables puedan estar bajo la influencia de factores socioeconómicos, demográficos, y de la salud de los niños/madre. Siendo estas variables: amamantamiento nocturno ( $<1$ año), uso de biberón-nocturno, y frecuencia de consumo de sacarosa entre comidas principales en la forma de jugo, fórmula, bocados y dulces. Las prácticas de la higiene oral pueden ser variables moderadoras de la asociación entre dieta y CIT, por tanto se incluyeron en el sexto-bloque: el inicio del cepillado dental, quien realiza el cepillado y el número de cepillados diarios. En el séptimo-bloque, la presencia de biopelícula visible se registró suponiendo que pueda estar bajo la influencia de la higiene, así como de las prácticas alimenticias. En el octavo-bloque: el nivel de SM salival se incluyó como una variable biológica más cercana al resultado, esto podría estar bajo la influencia de todas las variables de los bloques anteriores ${ }^{26}$.

Este estudio considerada a CIT una enfermedad social y ve como fortaleza evaluar una población con condiciones socioeconómicas homogéneas lo que favorecería la identificación de factores que estratifican el riesgo dentro de los grupos vulnerables y mejoran el control de los factores de confusión. Además, el modelo jerárquico les permitiría evaluar la mediación de factores más proximales sobre la asociación entre variables distales como situación socioeconómica y $\mathrm{CIT}^{26}$. 


\section{Conclusión}

Los modelos teóricos son construidos a fin de explicar la causalidad de los procesos biológicos, psicológicos y sociales que intervienen en el desarrollo de la enfermedad. Los modelos explicativos de CIT presentados dan cuenta de las similitudes y diferencias presentes a edades tempranas en la poblaciones estudiadas para dar predicción a la caries entre los niños con diversidad étnica o con deficiencias de salud bucal que devienen de las diferencias de culturas, propias de cada nación en el contexto mundial.

Todos los autores declaran que no existen conflictos potenciales de interés con respecto a la autoría y / o publicación de este artículo.

All authors declare no potential conflicts of interest with respect to the authorship and/or publication of this article

\section{Referencias}

1. Smith GA, Riedford K. Epidemiology of Early Childhood Caries: Clinical Application. Journal of Pediatric Nursing, 2013; 28: 369-373.

2. Helfenstein U, Steiner M, Menghini G. An outline of graphical Markov models in dentistry. Dent Health. 1999 Dec;16(4):220-6.

3. Sogaard, 1996 Sogaard A. Theories and models of health promotion. In: Schou L, Blinkhorn AS. (eds). Oral Health Promotion. Oxford: Oxford Medical Publications, 1996: 25-57.

4. Reisine S, Douglass JM. Psychological and behavioral issues in early childhood caries. Community Dent Oral Epidemiol 1998; 1(Suppl): 32-44.

5. Reisine S, Litt M, Tinanoff N. A biopsychosocial model to predict caries in preschool children.Pediatr Dent 1994; 16: 413-418.

6. Newton JT, Bower EJ. The social determinants of oral health: new approaches to conceptualizing and researching complex causal networks. Community Dent Oral Epidemiol 2005; 33: 25-34.

7. Boyce WT, Den Besten PK, Stamperdahl J, Zhan L, Jiang Y, Adler NE, Featherstone JD. Social inequalities in childhood dental caries: the convergent roles of stress, bacteria and disadvantage. Soc Sci Med. 2010, 71(9):1644-52.

8. Litt MD, Reisine S, Tinanoff N. Multidimensional causal model of dental caries development in lowincome preschool children. Public Health Reports 1995; 110:607-17.
9. Pine CM, Adair PM, Petersen PE, Douglass C, Burnside G, Nicoll AD, et al. Developing explanatory models of health inequalities in childhood dental caries. Community Dent Health 2004, 21(suppl 1):86-95.

10. Seow WK: Biological mechanisms of early childhood caries. Community DentOral Epidemiol 1998; 26: Supplement 1: 8-27.

11. Chestnutt I, Murdoch C, Robson K. Parents and carers'choice of drinks for infants and toddlers, inareas of social and economic disadvantage. Community Dent Health 2003;20:139-45.

12. Bhopal R. Is research into ethnicity and health racist, unsound, or important science? British Medical Journal 1997; 314, 1751-1756.

13. Bedi, R. The Oral Health of Minority Ethnic Communities in the United Kingdom. In: Assessing health needs of people from minority ethnic groups; 1998 ed. Rawaf, S. and Bahl, V. London: Royal College of Physicians.

14. Petersen, P.E. Social inequality in dental health towards theoretical explanations. Community Dentistry and Oral Epidemiology.1990, 18: 153-158.

15. Fisher-Owens SA, Gansky SA, Platt LJ et al. Influences on children's oral health: a conceptual model. Pediatrics 2007; 120: e510-e520.

16. Holgerson PL, Twetman S, Stecks.n-Blicks C. Validation of an agemodified caries risk assessment program (Cariogram) in preschool children. Acta Odontol Scand 2009, 67:106-112.

17. Téllez et al, 2013). Tellez M, Gomez J, Pretty I, Ellwood R, Ismail AI.Evidence on existing caries risk assessment systems: are they predictive of future caries? Community Dent Oral Epidemiol. 2013;41(1):67-78.

18. Bramlett MD, Soobader M-J, Fisher-Owens SA, Weintraub JA, Gansky SA, Platt LJ, Newacheck PW. Assessing a multilevel model of young children's oral health with national survey data. Community Dent Oral Epidemiol 2010; 38: 287-298.

19. Gao XL, Hsu CY, Xu Y, Hwarng HB, Loh T, Koh D. Building caries risk assessment models for children. $\mathrm{J}$ Dent Res 2010;89:637-43.

20. Boyce W.T., Den Besten P., Stamperdahl J et al., "Social inequalities in childhood dental caries: the convergent roles of stress, bacteria and disadvantage," Social Science and Medicine, 2010; 71 (9): 1644-1652.

21. Domejean S, White J, Featherstone J. Validation of the CDA CAMBRA caries risk assessment - a 6 year retrospective study. CDA Journal 2011;39:709-15.

22. MacRitchie HM, Longbottom C, Robertson M, Nugent Z, . Chan K, Radford JR, et al. Development of the Dundee Caries Risk Assessment Model (DCRAM)-risk model development using a novel 
application of CHAID analysis. Community Dent Oral Epidemiol 2012;40:37-45.

23. Seow KW. Environmental, maternal and child factors which contribute to early childhood caries: a unifying conceptual model. Int J Paed Dent 2012, 22:157-168.

24. Fierro Monti C, Pérez Flores M, Brunotto M.Simple predictive model for Early Childhood Caries of Chilean children. Rev Fac Cien Med Univ Nac Córdoba. 2014; 71(3):105-12.

25. OHughes S and Shewchuk RM. Child temperament, parent emotions, and perceptions of the child's feeding Experience.Intern $\mathrm{J}$ Behavioral Nutr and Physical Activity 2012, 9:64.

26. Nunes AM, da Silva AA, Alves CM, Hugo FN, Ribeiro

$\mathrm{CC}$.

Factors underlying the polarization of early childhoo $\mathrm{d}$ caries within a high-risk population. BMC Public Health. 2014 Sep 22; 14:988.

Correspondencia a/Corresponding to:

Claudia Fierro Monti Oral Pediatric Department,

University of Concepción,

Av. Roosevelt 1550, Concepción, 4030000 Chile.

Correo electrónico/E-mail: cfierro@udec.cl 